\title{
Ancillary Study
}

National Cancer Institute

\section{Source}

National Cancer Institute. Ancillary Study. NCI Thesaurus. Code C16041.

A study that is secondary to another study. 